\title{
Rare case of exogenous Candida dubliniensis endophthalmitis: a case report and brief review of the literature
}

Brian Douglas McMillan, Gary James Miller and John Nguyen*

\begin{abstract}
Background: Candida dubliniensis is a recently described opportunistic fungal pathogen that rarely infects the eye. Reported cases of $C$. dubliniensis endophthalmitis have been of endogenous etiology and demonstrated recovery of visual acuity with timely treatment. We herein report an unusual case of severe $C$. dubliniensis endophthalmitis requiring enucleation.
\end{abstract}

Findings: This is a retrospective, descriptive case report with a brief literature review. A 41-year-old Caucasian man, with a history of blunt trauma 8 months prior, presented to the emergency department with left eye pain and loss of vision 2 days after complicated cataract surgery. He was first evaluated by an outside ophthalmologist 3 months after trauma for left eye pain and progressive vision loss. He was found to have light perception vision with non-granulomatous anterior uveitis but no sign of ruptured globe. A dense cataract developed while he was treated with topical and subtenon's corticosteroids for which he underwent cataract surgery. Our examination revealed no light perception vision with a relative afferent pupillary defect, elevated intraocular pressure, moderate anterior chamber reaction, pupillary membrane, vitritis, and choroidal thickening on B-scan ultrasonography. Diagnostic vitrectomy revealed purulent vitreal debris, retinal detachment with severe retinal necrosis, and choroidal infiltrates. Operative fungal cultures grew C. dubliniensis. Despite intravitreal and systemic anti-fungal treatment, vision and pain did not improve, resulting in subsequent enucleation.

Conclusion: C. dubliniensis endophthalmitis is uncommonly encountered and typically has reasonable visual outcomes. This is the first reported case of $C$. dubliniensis, likely exogenous endophthalmitis, resulting in enucleation, illustrating the potential virulence of this newly described organism.

Keywords: Candida dubliniensis; Exogenous endophthalmitis; Enucleation; Ocular trauma

\section{Findings} Introduction

Fungal endophthalmitis can occur via either endogenous or exogenous route with Candida infections most commonly occurring from the endogenous route. Candida infections are predominately caused by Candida albicans species; however, several other species have been found to be pathologic especially in the immunocompromised state. Candida endogenous endophthalmitis often causes severe vision loss but rarely results in enucleation. In a study of 59 eyes with endogenous endophthalmitis, $56 \%$ of

\footnotetext{
* Correspondence: nguyenj@wvuhealthcare.com

Department of Ophthalmology, West Virginia University, 1 Stadium Dr, PO Box 9193, Morgantown, WV 26506-9193, USA
}

eyes with cultured positive yeast infection had a final visual acuity outcome of 20/200 or better, and only 3 progressed to enucleation, all of which were secondary to Aspergillus [1]. Exogenous fungal endophthalmitis is associated with poorer visual acuity outcomes and higher rates of enucleation given associated ocular trauma and direct inoculation of the eye; however, these cases are also most commonly caused by molds, fusarium, or fungal species other than Candida [2,3]. Candida dubliniensis is a relatively newly described species resulting in few reported cases of endogenous endophthalmitis, and most patients have reasonable visual acuity recovery [4-10]. We herein present an unusual case of severe C. dubliniensis endophthalmitis that required enucleation.

\section{实}




\section{Case report}

A 41-year-old Caucasian man presented to the emergency department with left eye pain and loss of vision after complicated cataract surgery 2 days prior. His past ocular history was significant for blunt trauma to the left eye with a stick while riding an all-terrain vehicle 8 months ago; over the following 3 months, the eye became painful with deterioration of vision for which he sought ophthalmic care. At that initial examination, outside records indicated that his vision was light perception, and there was significant conjunctival injection, corneal edema, and anterior chamber reaction precluding a view of the fundus. There was no recorded sign of previous ocular trauma, keratic precipitates, hypopyon, or afferent pupillary defect, and his poor vision was attributed to the inflammation from initial injury. He was treated for non-granulomatous anterior uveitis with topical and subtenon's corticosteroid injection for several months. The lens developed progressive cataract changes with corticosteroid treatment, and he underwent cataract surgery that was complicated and unsuccessful due to significant sequela from uveitic changes. An intraocular lens was not placed due to questionable lens capsule integrity.

Our examination revealed a visual acuity of 20/30 right eye (OD) and no light perception of the left eye (OS). The right pupil was reactive to light, but the left pupil was fixed and non-reactive, with a left relative afferent pupillary defect. He had marked restriction of ocular motility of the left eye due to pain. The left upper eyelid was ptotic and edematous. The orbit was slightly tense to retropulsion, but no proptosis was appreciated. The intraocular pressures were $19 \mathrm{mmHg}$ OD and $27 \mathrm{mmHg}$ OS.

Slit-lamp biomicroscopy of the left eye revealed injected and chemotic conjunctiva. The cornea was hazy with diffuse fluorescein staining of the epithelium. The cataract wound incision was intact, and Siedel test was negative. The anterior chamber was without hypopyon, but moderate cells and flare were seen. A pupillary membrane precluded view to the fundus of the left eye. The patient's vital signs were normal. Complete blood count revealed an elevated white blood cell count $(11,300$ cells per $\mathrm{dL})$ without a left shift.

B-scan ultrasonography of the left eye revealed diffuse vitritis and choroidal thickening. No retinal detachment was seen. The patient was admitted to the hospital for anterior chamber washout with removal of pupillary membrane, diagnostic vitrectomy, and intravitreal injection of vancomycin $(1 \mathrm{mg} / 0.1 \mathrm{ml})$, ceftazidime $(2.5 \mathrm{mg} / 0.1 \mathrm{ml})$, dexamethasone $(0.4 \mathrm{mg} / 0.1 \mathrm{ml})$, and amphotericin $B$ $(0.4 \mathrm{mg} / 0.1 \mathrm{ml})$. Intraoperative findings included significant fibrin anterior chamber reaction, purulent material filling the vitreous cavity, funnel retinal detachment, and multiple choroidal infiltrates. Retinal detachment repair was not attempted due to the poor status of the retinal tissue.
Initial gram stain revealed several polymorphonuclear cells with rare budding yeast and very rare pseudohyphae. Fungal culture subsequently revealed $C$. dubliniensis sensitive to amphotericin, caspofungin, and fluconazole (Figure 1). Serologic work-up for infection and inflammation included blood culture, rapid plasma reagin, fluorescent treponemal antibody-absorption, tuberculosis quantiferon gold, chest X-ray, angiotensin-converting enzyme, tick-borne disease panel, sedimentation rate, C-reactive protein, and human leukocyte antigen B27 which all were within normal limits.

The patient was treated with fluconazole $800 \mathrm{mg}$ daily and evaluated by the infectious disease service who found no signs of extra-ocular infection. He had a normal cardiac exam, and additional cardiac imaging was not recommended by the infectious disease service. He adamantly denied intravenous drug use but was found to be hepatitis $\mathrm{B}$ and $\mathrm{C}$ positive without prior history of vaccination. Human immunodeficiency virus testing was negative, and hemoglobin A1c was 5.6. The patient did not recover his vision and continued to experience severe pain for which enucleation surgery was offered. He underwent enucleation surgery with placement of a porous implant without complication. He was treated for 4 weeks with fluconazole and had an uneventful recovery at 3-month follow-up.

\section{Discussion}

Exogenous fungal endophthalmitis is usually caused by preceding fungal keratitis, trauma, and intraocular surgery, while endogenous fungal endophthalmitis is associated with recent hospitalization, systemic surgery, immunocompromised status, presence of intravenous lines, catheters, or intravenous drug use, organ transplant, and positive systemic cultures [11]. The mechanism of injury in this case is most likely due to a traumatic exogenous source in the setting of trauma. While he did not have

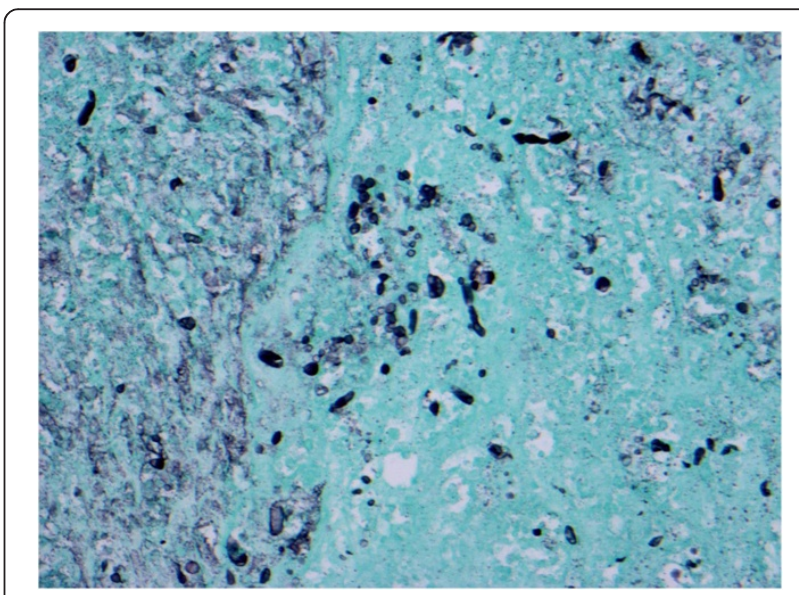

Figure 1 GMS stain illustrating hyphal elements. 
an open globe injury, the late presentation to the outside provider and the chronic use of corticosteroid may have delayed the diagnosis of chronic fungal endophthalmitis. The patient's hepatitis $\mathrm{B}$ and $\mathrm{C}$ infection placed him at increased risk for an endogenous infection; however, he did not have needle tracks on physical exam and a history of recent intravenous drug use along with a positive blood culture to entirely suggest an endogenous cause. Another potential exogenous source of infection is from the cataract extraction 2 days prior to presentation. While both the initial trauma and cataract surgery could result in the infection, the typical latency period of weeks to months (averaged 1.8 months) after initial traumatic fungal inoculation may suggest that the initial trauma being more likely. However, exogenous fungal endophthalmitis, rare in the acute post-operative period, has been shown to develop as early as within a day of inoculation [2].

Over $77 \%$ of exogenous fungal endophthalmitis cases are caused by molds with yeast as the second most common (23\%), specifically C. albicans as the most prevalent of the yeast species. There are, however, multiple species of Candida which can result in systemic infection in descending order of prevalence C. albicans (40\%), Candida glabrata (26\%), Candida parapsilosis (14\%), Candida tropicalis (6\%), Candida krusei (2\%), Candida lusitanie (1\%), and C. dubliniensis (1\%) [12]. Those typically associated with endophthalmitis include $C$. albicans and C. tropicalis $[1,11]$. C. dubliniensis is a rare and newly described organism initially discovered in 1995 in a population of acquired immunodeficiency syndrome patients in Dublin, Ireland [4]. It has been reported to be pathogenic primarily in immunocompromised patients, HIV, IV drug use, and neutropenic patients, and most isolated strains are susceptible to azoles, amphotericin B, and echinocandins [13]. However, in vitro studies have indicated that resistance to fluconazole can develop with long-term exposure due to overexpression of multidrug transporter proteins in some C. dubliniensis isolates [8], but has not been reported in clinical settings.

C. dubliniensis is closely related phenotypically with C. albicans but can be differentiated by poor growth of C. dubliniensis at elevated temperatures $\left(42^{\circ} \mathrm{C}\right.$ and $45^{\circ} \mathrm{C}$ ), chromographic culture techniques illustrated by CHROMagar Candida media (DRG International Inc., Springfield, NJ, USA) resulting in growth of dark green C. dubliniensis colonies and more specifically by speciesspecific polymerase chain reaction-enzyme immunoassay techniques [14]. These new technologies are helping to make the diagnosis of $C$. dubliniensis infections more readily with a subsequent increase in reported cases. The first case of endogenous $C$. dubliniensis endophthalmitis was presented in 2008 in a healthy 38 -year-old male without immunosuppression or intravenous use history [5].
Pelegrin et al. subsequently described another case of endogenous C. dubliniensis endophthalmitis in a 41-yearold HIV-infected intravenous drug abuser in Spain in 2009 with good recovery of vision from 20/400 to 20/80. Espinosa-Heidmann et al. also reported a case of endogenous C. dubliniensis endophthalmitis in a 27-year-old man without immunosuppression with similar recovery of visual acuity after treatment. Moloney and Park described an additional five cases of $C$. dubliniensis endogenous endophthalmitis in patients with a history of IV drug use and hepatitis C. Four patients had final visual outcome of 20/60 or better, and one had hand motion vision. A last recent case published by Rosenberger et al. again illustrated excellent visual outcome of 20/25. While $C$. dubliniensis becomes an established, although rare, causative agent of endogenous fungal endophthalmitis, there has been no reported case of exogenous etiology nor a severe case resulting in enucleation upon our review.

In summary, we report the first case of $C$. dubliniensis endophthalmitis leading to eventual enucleation and likely the first case of exogenous etiology. While C. dubliniensis endogenous endophthalmitis cases have reasonable visual outcome, C. dubliniensis endophthalmitis can progress to cause severe intraocular destruction with complete loss of vision that requires enucleation, particularly in exogenous cases.

\section{Abbreviations}

C. dubliniensis: Candida dubliniensis; OD: right eye; OS: left eye; NLP: no light perception.

\section{Competing interests}

The authors declare that they have no competing interests.

\section{Authors' contributions}

BDM participated in obtaining clinical data, review of the literature, and drafting of the manuscript. GJM participated in the critical management in patient care and drafting of the manuscript. JN participated in the critical management in patient care and assisted in the literature review and drafting of the manuscript. All authors read and approved the final manuscript.

\section{Acknowledgements}

The authors acknowledge Matthew Szarko, MD from the West Virginia University Pathology Department. This project received support from an Unrestricted Challenge Grant from Research to Prevent Blindness to WVU.

Received: 27 February 2014 Accepted: 17 April 2014 Published: 2 May 2014

\section{References}

1. Lingappan A, Wykoff CC, Albini TA (2012) Endogenous fungal endophthalmitis: causative organisms, management strategies, and visual acuity outcomes. Am J Ophthalmol 153:162-166

2. Wykoff CC, Flynn HW, Miller D, Scott IU, Alfonso EC (2008) Exogenous fungal endophthalmitis: microbiology and clinical outcomes. Ophthalmology 115:1501-1507

3. Pflugfelder SC, Flynn HW, Zwickey TA, Forster RK, Tsiligianni A, Culbertson WW, Mandelbaum S (1988) Exogenous fungal endophthalmitis. Ophthalmology 95:19-30

4. Sullivan DJ, Westermeng TJ, Haynes KA, Bennett DE, Coleman DC (1995) Candida dubliniesis sp. nov.: phenotypic and molecular characterization of a novel species associated with oral candidosis in HIV-infected individuals. Microbiology 141:1507-1521 
5. Sedeek RW, Shah M, Gentile R, Samson CM (2008) First case report of Candida dubliniensis endogenous endophthalmitis. ARVO 952:D782

6. Espinosa-Heidmann DG, McMillan BD, Lasala PR, Stanley J, Larzo CR (2012) Candida dubliniensis endophthalmitis: first case in North America. Int Ophthalmol 32:41-45

7. Pelegrin L, Mesquida M, Adan A, Cervera C, Bosch-Mestres J, Esteban-Redondo C Juiz-Gonzales P, Llorec V, Miro JM (2011) Candida dubliniensis endophthalmitis in a HIV-infected intravenous drug abuser. Mycoses 54:856-858

8. Moran GP, Sanglard D, Donnelly SM, Shanley DB, Sullivan DJ, Coleman DC (1998) Identification and expression of multidrug transporters responsible for fluconazole resistance in Candida dubliniensis. Antimicrob Agents Chemother 42:1819-1830

9. Rosenberger E, Youssef DA, Safdar S, Larzo CR, Myers J (2013) Third case of Candida dubliniensis endogenous endophthalmitis in North America: case report and review of the literature. Int Ophthalmol. doi:10.1007/s10792-013-9880x

10. Moloney TP, Park J (2013) Candida dubliniensis endophthalmitis: five cases over 15 years. J Ophthalmic Inflamm Infect 3:66

11. Sridhar J, Flynn HW, Kuriyan AE, Miller D, Albini T (2013) Endogenous fungal endophthalmitis: risk factors, clinical features, and treatment outcomes in mold and yeast infections. J Ophthalmic Inflamm Infect 3:60

12. Yin KK, Levine BM (2012) Ocular involvement among inpatients with fungemia: analysis from a New York City tertiary care center. Invest Ophthalmol Vis Sci 53, E-Abstract 1682. http://abstracts.iovs.org/cgi/content/short/53/6/1682

13. Pfaller MA, Messer SA, Gee S, Joly S, Pujol C, Sullivan DJ, Coleman DC, Soll DR (1999) In vitro susceptibilities of Candida dubliniensis isolates tested against the new triazole and echinocandin antifugal agents. J Clin Microbiol 37:870-872

14. Ellepola AB, Hurst SF, Elie CM, Morrison CJ (2003) Rapid and unequivocal differentiation of Candida dubliniensis from other Candida species using species-specific DNA probes: comparison with phenotypic identification methods. Oral Microbiol Immunol 18:379-388

doi:10.1186/1869-5760-4-11

Cite this article as: McMillan et al:: Rare case of exogenous Candida dubliniensis endophthalmitis: a case report and brief review of the literature. Journal of Ophthalmic Inflammation and Infection 2014 4:11.

\section{Submit your manuscript to a SpringerOpen ${ }^{\circ}$ journal and benefit from:}

- Convenient online submission

- Rigorous peer review

- Immediate publication on acceptance

- Open access: articles freely available online

- High visibility within the field

- Retaining the copyright to your article

Submit your next manuscript at $\gg$ springeropen.com 